\title{
Homozygous missense and nonsense mutations in BMPR1B cause acromesomelic chondrodysplasia-type Grebe
}

\author{
Luitgard M Graul-Neumann ${ }^{1,11}$, Alexandra Deichsel ${ }^{2,3,11}$, Ulrike Wille ${ }^{2,3}$, Naseebullah Kakar ${ }^{4,5}$, Randi Koll ${ }^{6}$, \\ Christian Bassir ${ }^{7}$, Jamil Ahmad ${ }^{5}$, Valerie Cormier-Daire ${ }^{8}$, Stefan Mundlos ${ }^{2,6}$, Christian Kubisch ${ }^{4}$, \\ Guntram Borck ${ }^{4}$, Eva Klopocki ${ }^{6,9}$, Thomas D Mueller ${ }^{10}$, Sandra C Doelken ${ }^{\star, 6}$ and Petra Seemann ${ }^{2,3}$
}

Acromesomelic chondrodysplasias (ACDs) are characterized by disproportionate shortening of the appendicular skeleton, predominantly affecting the middle (forearms and forelegs) and distal segments (hands and feet). Here, we present two consanguineous families with missense (c.157T >C, p.(C53R)) or nonsense (c.657G > A, p.(W219*)) mutations in BMPR1B. Homozygous affected individuals show clinical and radiographic findings consistent with ACD-type Grebe. Functional analysis of the missense mutation C53R revealed that the mutated receptor was partially located at the cell membrane. In contrast to the wild-type receptor, C53R mutation hindered the activation of the receptor by its ligand GDF5, as shown by reporter gene assay. Further, overexpression of the C53R mutation in an in vitro chondrogenesis assay showed no effect on cell differentiation, indicating a loss of function. The nonsense mutation (c.657G $>$ A, p.(W219*)) introduces a premature stop codon, which is predicted to be subject to nonsense-mediated mRNA decay, causing reduced protein translation of the mutant allele. A lossof-function effect of both mutations causing recessive ACD-type Grebe is further supported by the mild brachydactyly or even non-penetrance of these mutations observed in the heterozygous parents. In contrast, dominant-negative BMPR1B mutations described previously are associated with autosomal-dominant brachydactyly-type A2.

European Journal of Human Genetics (2014) 22, 726-733; doi:10.1038/ejhg.2013.222; published online 16 October 2013

Keywords: BMPR1B; acromesomelic chondrodysplasia; Grebe syndrome; genital development

\section{INTRODUCTION}

Osteochondrodysplasias are a clinically and genetically heterogeneous group of hereditary skeletal disorders. ${ }^{1}$ Among these, acromesomelic chondrodysplasias (ACDs) constitute a rare subgroup characterized by short stature, severely shortened limbs, and hand/foot malformations. The severity of limb abnormalities increases in a proximo-to-distal gradient with hands and feet being most severely affected, showing brachydactyly and/or rudimentary fingers. The group of ACDs are classified into five subgroups according to the Nosology and Classification of Genetic Skeletal Disorders: ${ }^{2}$ Acromesomelic dysplasia-type Maroteaux (MIM 602875), Grebe dysplasia (Grebe type, MIM 200700 and Hunter Thompson type, MIM 201250), Fibular hypoplasia and complex brachydactyly (Du Pan, MIM 228900), acromesomelic dysplasia with genital anomalies (MIM 609441) and Acromesomelic dysplasia Osebold-Remondini type (MIM 112910). Grebe dysplasia and $\mathrm{Du}$ Pan syndrome share an autosomal recessive inheritance pattern and are caused by homozygous or compound heterozygous mutations in growth and differentiation factor 5 (GDF5; MIM $\left.{ }^{*} 601146\right)$.
GDF5 is a member of the TGF- $\beta$ superfamily belonging to the subgroup of bone morphogenetic proteins (BMPs). GDF5 binds extracellular to transmembrane serine threonine kinases such as BMPR1A and BMPR1B, with a preference for BMPR1B. ${ }^{3}$ All reported mutations in GDF5 associated with ACD are caused by a homozygous functional loss of GDF5, which is caused by either deletions, duplications, or insertions leading to a premature translational stop or caused by point mutations targeting the cleavage site or the mature domain of GDF5, thereby compromising its regular function. $^{4-13}$ Interestingly, one individual with a homozygous mutation in BMPR1B has been reported presenting with a subtype of ACD with additional genital anomalies, ${ }^{14}$ implicating that mutations in the ligand and its receptor can cause similar phenotypes. Heterozygous carriers of dominant-negative mutations in BMPR1B are usually affected by brachydactyly-type A2 (BDA2; MIM number 112600), which is characterized by a shortened middle phalanx of the index finger and variable clinodactyly of the fifth finger. ${ }^{15}$ In contrast, carriers of heterozygous mutations in the ligand, GDF5, are usually affected by brachydactyly-type C (MIM number $113100),{ }^{16,17}$ a condition characterized by brachymesophalangy of

${ }^{1}$ Ambulantes Gesundheitszentrum der Charité-Universitätsmedizin Berlin, Berlin, Germany; ${ }^{2}$ Berlin-Brandenburg Center for Regenerative Therapies (BCRT), CharitéUniversitätsmedizin Berlin, Berlin, Germany; ${ }^{3}$ Berlin-Brandenburg School for Regenerative Therapies (BSRT), Charité- Universitätsmedizin Berlin, Berlin, Germany; ${ }^{4}$ Institute of Human Genetics, University of UIm, Ulm, Germany; ${ }^{5}$ Department of Biotechnology and Informatics, BUITEMS, Quetta, Pakistan; ${ }^{6}$ Institute for Medical and Human Genetics, Charité- Universitätsmedizin Berlin, Berlin, Germany; ${ }^{7}$ Pediatric Radiology, Charité—Universitätsmedizin Berlin, Berlin, Germany; ${ }^{8}$ Department of Genetics, Paris DescartesSorbonne Paris Cité, Fondation Imagine, Hopital Necker-Enfants Malades, Paris, France; ${ }^{9}$ Institute for Human Genetics, University of Würzburg, Würzburg, Germany; 10Julius-von-Sachs Institute, University of Würzburg, Würzburg, Germany

${ }^{11}$ These authors contributed equally to this work.

*Correspondence: SC Doelken, Institute for Medical and Human Genetics, Charité-Universitätsmedizin Berlin, Augustenburger Platz 1, 13353 Berlin, Germany. Tel: +49 30450569116 or +49 30450569 132; Fax: +49 30450569 914; E-mail: sandra.doelken@charite.de

Received 30 May 2013; revised 28 August 2013; accepted 30 August 2013; published online 16 October 2013 
index, middle, and little fingers as well as shortening of the first metacarpal. Ring fingers are only mildly affected and hyperphalangy of the index and middle fingers might occur. ${ }^{9,18-20}$ However, clinical phenotypes resulting from heterozygous $B M P R 1 B$ and GDF5 mutations are variable and also show overlapping features. ${ }^{21,22}$

Here we present two consanguineous families segregating as yet unreported mutations in BMPR1B (c.157T $>$ C, p. $(\mathrm{C} 53 \mathrm{R})$ and (c.657G $>$ A, p. $\left(\mathrm{W} 219^{*}\right)$ ) with a total of three affected individuals carrying the respective mutations in a homozygous state. Both mutations are proposed to cause a loss of function of BMPR1B.

\section{MATERIALS AND METHODS}

\section{Clinical investigation and molecular analyses}

All clinical investigations were conducted in accordance with the Declaration of Helsinki principles. Informed consent was obtained for genetic analysis. Genomic DNA was extracted from peripheral blood samples by standard methods. With regard to the ACD phenotype, we first performed mutation screening of GDF5 (NM_000557.2) and subsequently of BMPR1B (NM_001256794.1). The coding regions of GDF5 and BMPR1B as well as the flanking intronic sequences were amplified by standard PCR protocols. The primer sequences and PCR conditions for the molecular testing can be found elsewhere $\left(G D F 5{ }^{23} B M P R 1 B^{15}\right)$. Sequencing was done using the ABI Prism BigDye Terminator Sequencing Kit (Applied Biosystems, Life Technologies, Foster City, CA, USA) with PCR primers used as sequencing primers. Products were evaluated on an automated capillary sequencer (Applied Biosystems 3730).

Obtained sequence variant information was submitted to the Leiden Open Variation Database (https://www.grenada.lumc.nl/LOVD2/mendelian_genes/ home.php?select_db=BMPR1B).

\section{Sequence alignment and structural data}

Amino-acid sequences of the ligand-binding domain (LBD) of the seven human TGF- $\beta$-type I receptors as well as chicken and mouse orthologs of BMPR1B were aligned using Clustal Omega ${ }^{24,25}$ (http://www.ebi.ac.uk/ Tools/services/web/toolform.ebi?tool=clustalo) and colored using CHROMA ${ }^{26}$ (http://www.llew.org.uk/chroma/).

Modeling of the BMPR1B-mutant C53R was performed using the BMPR1B coordinates of the crystal structure of GDF5 bound to wild-type BMPR1B (PDB entry 3EVS). ${ }^{27}$ First, the disulfide bond involving cysteine residues 32 and 53 of BMPR1B was removed and C53 was replaced by an arginine residue using the ProteinDesign tool of the software package Quanta2008 (Accelrys, Inc., San Diego, CA, USA). The resulting model of C53R was then refined by energy minimization using the all-hydrogen force field of CHARMM27 and employing only geometrical energy terms. As the charged side chain of the introduced arginine at position 53 is almost completely buried inside the hydrophobic core of the BMPR1B, further energy minimization and short $(50 \mathrm{ps})$ in vacuo molecular dynamics simulations were performed using the CHARMM27 force field described above but employing also electrostatic energy terms using a distance-dependent treatment of the coulomb term. Images of the three-dimensional structure of a BMPR1B-GDF5 complex were based on the PDB-file $3 \mathrm{EVS}^{27}$ rendered by PyMOL Molecular Graphics System, Schrödinger, LLC (Portland, OR, USA).

\section{Cloning of expression constructs}

The coding sequences of murine Bmprlb and human GDF5 were cloned into the shuttle vector pSLAX13. ${ }^{28}$ Murine Bmprlb in pSLAX13 was used as a template for in vitro mutagenesis to introduce the homologous mutation C53R, to delete the LBD (dLBD), or to insert a hemagglutinin (HA) tag. Primers for mutagenesis are available in Supplementary Table 1. Inserts were subcloned into the expression vector pCS2 + via the ClaI restriction site.

\section{$\mathrm{NIH} / 3 \mathrm{~T} 3$ cultivation}

NIH/3T3 cells (American Type Culture Collection (ATCC), Manassas, VA, USA) were cultivated in DMEM $4.5 \mathrm{~g} / 1$ glucose (Lonza, Verviers, Belgium) supplemented with 10\% FBS superior (Biochrom AG, Berlin, Germany), 2 mM
L-glutamine (Lonza) and penicillin/streptomycin (Lonza) in a humidified atmosphere of $5 \% \mathrm{CO}_{2}$ at $37^{\circ} \mathrm{C}$.

\section{Immunocytochemistry}

NIH/3T3 cells were seeded in black 'CellCarrier' 96-well plates with an optically clear bottom (Perkin Elmer, Waltham, MA, USA) and transfected with HA-tagged Bmprlb-constructs in pCS2 + using Lipofectamine 2000 (Invitrogen, Life Technologies, Carlsbad, CA, USA). Twenty-four hours after transfection, cells were washed twice and incubated for $1 \mathrm{~h}$ at $37^{\circ} \mathrm{C}$ in serumfree DMEM $4.5 \mathrm{~g} / \mathrm{l}$ glucose (Lonza). After washing with PBS, cells were fixed for $15 \mathrm{~min}$ at room temperature (RT) in $4 \%$ paraformaldehyde in PBS and washed three more times with PBS. For permeabilization, cells were incubated for $15 \mathrm{~min}$ in PBS containing $0.2 \%$ Triton-X-100. To block nonspecific binding, cells were incubated in 10\% FBS superior (Biochrom AG) in PBS overnight at $4{ }^{\circ} \mathrm{C}$. This was followed by incubation with rabbit anti-HA antibody (H6908, Sigma-Aldrich, St Louis, MO, USA) diluted 1:100 in PBS containing 10\% FBS superior (Biochrom AG) at RT for $1 \mathrm{~h}$. Detection was achieved after extensive washing by incubation with anti-rabbit-Alexa Fluor 488 antibody (A11008, Molecular Probes Life Technologies, Eugene, OR, USA) diluted 1:2000 in PBS containing 10\% FCS (Biochrom AG) and DAPI (Invitrogen, Life Technologies). Fluorescence images were recorded with the 'Operetta' High Content Imaging System (Perkin Elmer) using $\times 60$ magnification. Wide-field images were deconvolved by iterative restoration ( 25 steps) using Volocity 3D Image Analysis Software (Perkin Elmer).

\section{Luciferase reporter gene assay}

$\mathrm{NIH} / 3 \mathrm{~T} 3$ cells were seeded in a 96-well plate and co-transfected using Lipofectamine 2000 (Invitrogen, Life Technologies) at about 70\% confluency with pCS2 + expression constructs of Bmpr1b ${ }^{\mathrm{wt}}, B m p r 1 b^{\mathrm{C} 53 \mathrm{R}}, B m p r 1 b^{\mathrm{dLBD}}$, GDF5, BRE luciferase reporter construct ${ }^{29}$ and the Renilla luciferase vector pRL-TK (Promega, Madison, WI, USA), which served as an internal control for transfection efficiency. Cells were cultivated in DMEM $4.5 \mathrm{~g} / \mathrm{l}$ glucose (Lonza) supplemented with 1\% FBS superior (Biochrom AG), $2 \mathrm{~mm}$ L-glutamine (Lonza), penicillin/streptomycin (Lonza) for $42 \mathrm{~h}$ after transfection and subsequently collected and lyzed in potassium phosphate buffer ( $9 \mathrm{~mm}$ potassium dihydrogen phosphate, $91 \mathrm{~mm}$ dipotassium phosphate, $0.2 \%$ Triton-X-100). Dual luciferase activity was determined as described previously $^{30}$ using Mithras LB 940 (Berthold Technologies GmbH \& Co. KG, Bad Wildbad, Germany).

\section{Micromass cultures}

The coding sequence of chicken BMPR1B in pSLAX13. ${ }^{15}$ was used as a template for in vitro mutagenesis to introduce the homologous mutation C53R and dLBD. Primers for mutagenesis are available in Supplementary Table 1. Inserts were subcloned into the avian-specific retroviral construct RCASBP(A) via ClaI. ${ }^{31}$

Production and concentration of viral supernatant was performed as described. ${ }^{32}$ Fertilized chicken eggs (VALO BioMedia GmbH, OsterholzScharmbeck, Germany) were incubated at $37.5^{\circ} \mathrm{C}$ in a humidified egg incubator for $\sim 4.5$ days. Micromass cultures were isolated from limb buds of Hamburger Hamilton stage $23-24^{33}$ as described, with minor modifications. ${ }^{15}$ Micromass cultures were plated at a density of $2 \times 10^{5}$ cells per $12 \mu \mathrm{l}$ drop in the middle of a 24 -well tissue-culture plate. Infection was performed with a titer of $1 \times 10^{7}$ plaque-forming units of the concentrated RCAS viral supernatants, as described. ${ }^{28}$ Empty RCAS virus was used as a control. Cells were allowed to attach for $2 \mathrm{~h}$ in a humidified atmosphere of $5 \%$ $\mathrm{CO}_{2}$ at $39^{\circ} \mathrm{C}$ and then complemented with DMEM-F12 (Biochrom AG) culture medium supplemented with 10\% FBS superior (Biochrom AG), $0.2 \%$ chicken serum (Sigma-Aldrich), L-glutamine (Lonza), penicillin/streptomycin (Lonza). The culture medium was replaced every 2 days. Micromass cultures were washed twice with PBS, fixed with Kahles' Fixative (28.9\% ethanol, $0.37 \%$ formaldehyde, $3.9 \%$ acetic acid), and stained with $0.05 \%$ Alcian Blue (SigmaAldrich) in $0.1 \mathrm{M} \mathrm{HCl}$ at day 7. Quantification of the staining was achieved by extraction with $6 \mathrm{M}$ guanidine hydrochloride (Carl Roth $\mathrm{GmbH}+\mathrm{Co}$. KG, Karlsruhe, Germany) overnight at RT. Dye concentration was determined spectrophotometrically at $595 \mathrm{~nm}$. 


\section{RESULTS}

\section{Family I}

We report on a 2-year-old female (Figure 1Aa) whose Lebanese parents are healthy first cousins. The first child of the couple died shortly after birth, probably because of an infectious disease. Both parents are of normal height (mother: $163 \mathrm{~cm}$, father: $173 \mathrm{~cm}$ ). The family history is otherwise unremarkable (pedigree scheme in Figure 1Ab).

The patient presented with disproportionate short stature (height: $76 \mathrm{~cm}-4 \mathrm{SD}$ ) because of severe acromesomelic limb shortening (Figure 1Aa). The trunk was of normal length. Her hands showed marked shortening of digits and pronounced radially deviation of fingers. All fingernails were present (Figure 1Ac-f). Dimpling of the skin was present over the anterior aspect of the shin, at the apex of the site of anteromedial bowing. The fibulae were not palpable. Valgus instability of the ankle joints was evident. The feet were small and broad. All toes were short and functionless with constrictions at their bases and all toenails were present (Figure $1 \mathrm{Ag}$ and $\mathrm{h}$ ).

On X-ray examination, pelvis and femora appeared normal. Tibiae were shortened and severely bowed, and fibulae were absent resulting in bilateral dislocation of the tibiotalar joints (Figure 1Aj). Bilateral shortened ulnae, mildly bowed radii, and small ossification centers of the radial epiphyses were observed. One ossified carpal bone was detected (Figure 1Am and $\mathrm{n}$ ). The patient had absent 1st metacarpals, slender 2nd metacarpals, clumsy and shortened 3rd-5th metacarpals

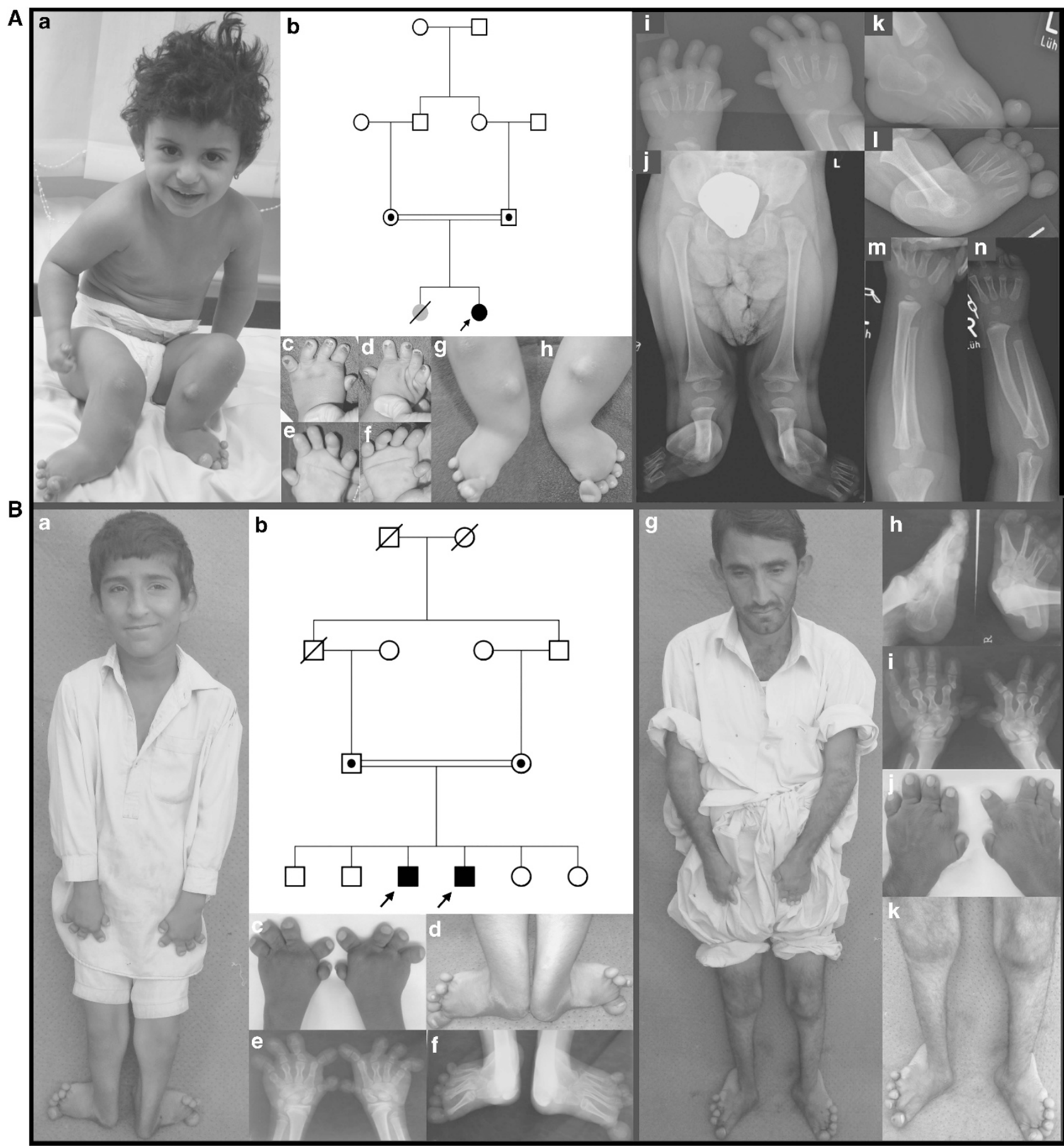


as well as absence of the proximal thumb phalanx and the proximal and middle phalanges of the 5th fingers. Fingers 2-4 appeared to have only two phalangeal bones with very small proximal phalanges (Figure 1Ai). In the feet, talus, calcaneus, and possible ossification centers of the ossa navicularia were visible, all metatarsals were present, and all distal phalanges of the feet were hypoplastic. On the left side, very small ossification centers of the phalanges of the 3rd and 4 th toe were visible (Figure $1 \mathrm{Ak}$ and $\mathrm{l}$ ).
Ultrasound examination of the abdomen showed a normal uterus; the ovaries could not be evaluated because of bowel gas superposition. Ultrasound examination of the heart was normal. Hearing and ophthalmologic examination were also normal.

The mother showed mild BDA2 with shortened middle phalanges of the 2nd and 5th fingers and toes (Supplementary Figure 1Aa-c). The father did not show any abnormalities (Supplementary Figure 1Ad-f).

Table 1 Clinical features of the affected individuals of the families with homozygous mutations in BMPR1B (c.157T $>$ C, p.(C53R); c.657G >A, p.(W219*); c.361_368del 5'-GGACCTAT-3'14)

\begin{tabular}{|c|c|c|c|c|c|}
\hline \multirow[b]{2}{*}{ Feature } & \multirow[b]{2}{*}{$H P O: I D$} & \multirow{2}{*}{$\begin{array}{c}c .157 T>C, p .(C 53 R) \\
\text { \& } 2 \text { years }\end{array}$} & \multicolumn{2}{|c|}{ c. $657 G>A, p .\left(W 219^{*}\right)$} & \multirow{2}{*}{$\begin{array}{c}\text { c.361_368delGGACCTAT } \\
\text { \& } 16 \text { years }\end{array}$} \\
\hline & & & o 15 years & o 19 years & \\
\hline Short stature & HP:0004322 & + & + & + & + \\
\hline Acromesomelia & HP:0003086 & + & + & + & + \\
\hline Brachydactyly & HP:0001156 & + & + & + & + \\
\hline Radial deviation of fingers & HP:0009466 & + & + & + & + \\
\hline Aplasia of the 1st metacarpal & HP:0010035 & + & + & + & + \\
\hline Short 2nd metacarpal & HP:0010038 & + & + & + & - \\
\hline Short 3rd metacarpal & HP:0010041 & + & + & + & - \\
\hline Short 4th metacarpal & HP:0010044 & + & + & + & - \\
\hline Short 5th metacarpal & HP:0010047 & + & + & + & - \\
\hline Absent proximal phalanx of thumb & HP:0009637 & + & - & - & + \\
\hline Aplasia of the proximal phalanx of the 5 th finger & HP:0009225 & + & + & - & - \\
\hline Absent middle phalanx of 5 th finger & HP:0009162 & + & - & - & - \\
\hline Aplasia of the proximal phalanx of the 2 nd finger & HP:0009596 & + & + & + & + \\
\hline Abnormally shaped carpal bones & HP:0006014 & * & + & + & + \\
\hline Carpal synostosis & HP:0009702 & * & + & + & + \\
\hline Carpometacarpal synostosis & HP:0100328 & * & - & + & - \\
\hline Tarsal synostosis & HP:0008368 & - & - & - & + \\
\hline Shortening of all distal phalanges of the toes & HP:0005793 & + & + & + & + \\
\hline Hypoplasia of the ulna & HP:0003022 & + & + & + & + \\
\hline Radial bowing & HP:0002986 & + & - & - & - \\
\hline Tibial bowing & HP:0002982 & + & - & - & - \\
\hline Hypoplastic tibia & HP:0005736 & + & + & + & + \\
\hline Fibular aplasia & HP0002990 & + & + & + & + \\
\hline Skin dimple over apex of long bone angulation & HP:0001024 & + & - & - & - \\
\hline Talipes equinovarus & HP:0001762 & + & + & + & + \\
\hline Aplasia of the ovary & HP:0010463 & NA & l & l & + \\
\hline Hypoplasia of the uterus & HP:0000013 & - & l & l & + \\
\hline
\end{tabular}

Abbreviations: +, present; -, absent; *, not yet possible to evaluate because of age of patient (only one carpal bone present); NA, not evaluated because of bowel gas superposition and age of patient; /, not applicable because of male sex.

The features are coded using terms from the Human Phenotype Ontology. ${ }^{37}$

Figure 1 Homozygous BMPR1B mutations cause ACD. (Aa) Affected 2-year-old girl from family A: phenotype with short stature and acromesomelic limb shortening. (Ab) Pedigree of family A with segregating missense mutation c.157T $>$ C, p.(C53R). (Ac-f) The hands show severe brachydactyly of all digits and radially deviated fingers. (Ag, $\mathbf{h}$ ) The feet show clubfoot deformity with knob-like toes and there is dimpling of the skin over the anterior aspect of the shin, at the apex of the site of anteromedial bowing, the feet are small and broad, all toes are short and functionless with constrictions at their bases, and all toenails are present. (Ai) Hand radiographs show only one carpal bone, the 1st metacarpals are absent, 2nd-5th metacarpals are hypoplastic, the proximal thumb phalanx and the proximal and middle phalanges of the 5th fingers are absent, and fingers 2-4 appear to have only two phalangeal bones (Aj) Radiographs of the legs display shortened bowed tibiae and fibular aplasia, as well as bilateral dislocation of the tibiotalar joints. (Ak and I) In the feet, talus, calcaneus, and possible ossification centers of the ossa navicularia are visible, all metatarsals are present, all distal phalanges of the feet are hypoplastic, and very small ossification centers of the phalanges of the 3rd and 4th toe are visible. (Am, $\mathbf{n}$ ) Radiographs of the arms show bilateral shortened ulnae, the radii appear mildly bowed. (Ba) Affected 15-year-old boy from family B: phenotype with short stature and acromesomelic limb shortening. (Bb) Pedigree of family B with segregating nonsense mutation c.657G $>$ A, p.(W219*). (Bc, j) The hands show marked shortening of digits and deviated fingers, all fingernails are present. (Bd, k) The feet are small and broad, all toes are short and functionless with constrictions at their bases, and all toenails are present. (Be, i) Bilateral shortened ulnae, deformed carpal bones with carpal fusions, shortened metacarpals with absence of 1 st metacarpals, short and deformed proximal and middle phalanges of the fingers, absence of the proximal phalanges of the index fingers; in the thumb, a small proximal phalanx is present. (Be) Absence of the proximal phalanx of the 5th finger. (Bi) Fusions of carpal bones with the $2 \mathrm{nd}$ and $3 \mathrm{rd}$ metacarpals. (Bf, $\mathbf{h}$ ) In the feet, talus, calcaneus, and possible remnants of some ossa navicularia are visible, all metatarsals are present, all distal phalanges of the feet are hypoplastic, and some mislocalized middle or proximal phalanges seems to be present. (Bg) Affected 19-year-old boy from family B: phenotype with short stature and acromesomelic limb shortening. 


\section{Family II}

The second family originates from Pakistan; the parents are healthy first cousins and the two affected brothers are aged 15 (Figure 1Ba) and 19 years (Figure $1 \mathrm{Bg}$ ), respectively. Both parents presented with short stature (mother: $150 \mathrm{~cm}$; father: $145 \mathrm{~cm}$ ). The family history is otherwise unremarkable (pedigree Figure $1 \mathrm{Bb}$ ).

The patients also presented with disproportionate short stature because of severe acromesomelic limb shortening (Figure 1Ba and g);

a
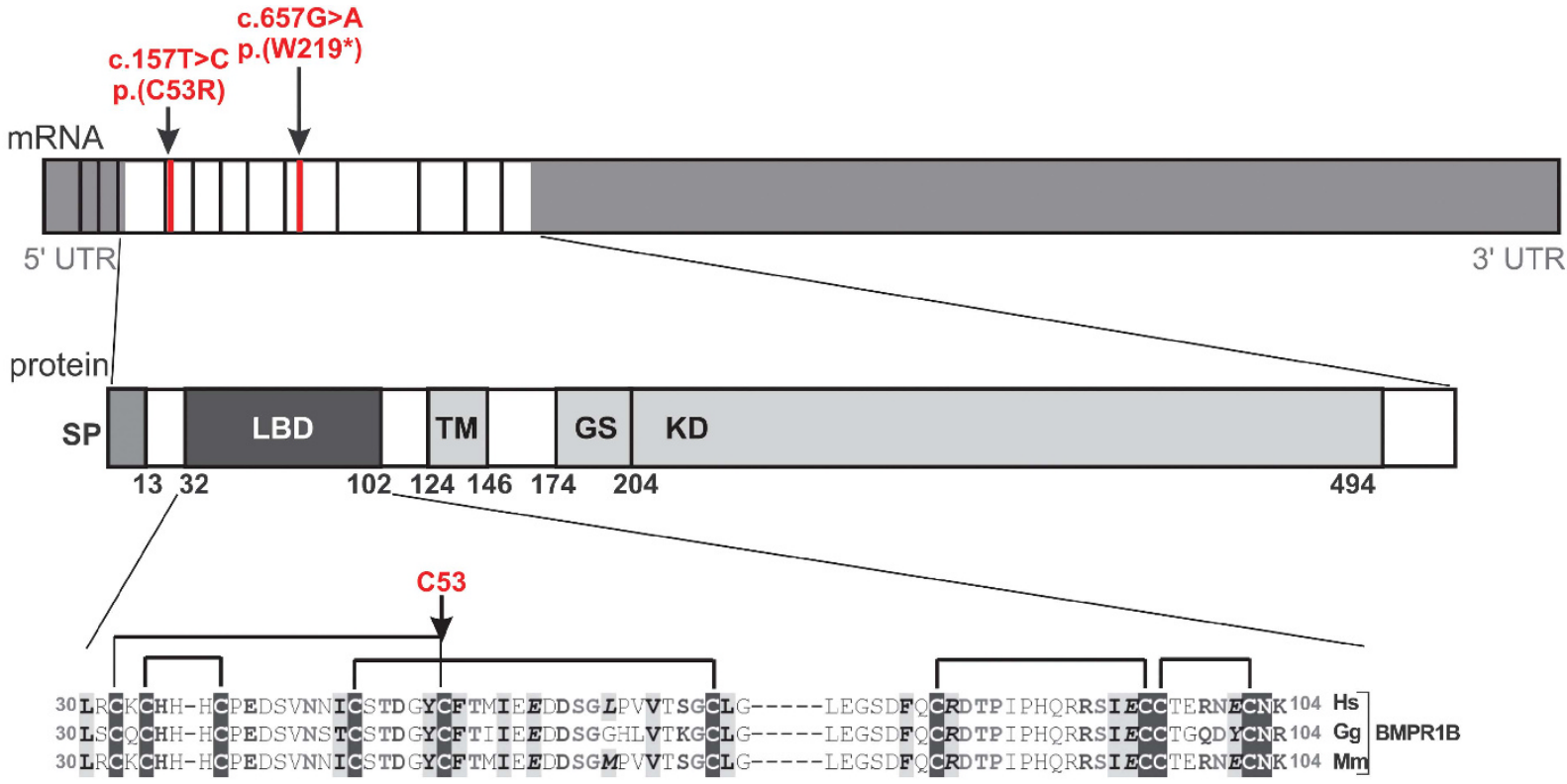

32VTCTCESPICK----GP TCRGA-WCTVVLVREEGRHPQEHRGC-------GNLHRELCRGRPT--EFVNHYCC-DSIILNH 97 ACVRL1

33YMCVCEGLSCGN---EDHCEGQ-OCFSSLS I-NDG FHVYOKGCF QV----YEQGKMTCKTPP--SPGQAVECC-QGDWCNR 101 ACVR1

59 L KCY CS G-HCPD DAINN TC I TNGHCFAI I E EDDQGET T LASGCMK-----YEGSDF CKD SPKAQLRRTI ECC-RTN LCNQ 132 BMPR1A

32L LCACT--SCLQ--ANY TCETD GACMVSI FN-LD GMEHHVRTCI PKVELVPAGKPFYCLSSE---DLRNTHCC-Y TD YCNR 103 ACVR1B

34 L CECH--LCTK--DNE TCVTD GLCFVSVT E-TTD KVI HNSMCI A I I DLI PRDRPFVCAPSS KTGSVTT T YCC-NQD HCNK 108 TGFBR1

26 LKCVCL--LCDS--SNE TCQTEGACWASVML-TNG KE QVI KSCV---SLPELNA VVECHSSN---NVTKTECC-ETDFCNN 94 ACVR1C

l.C.Cp...C.p...s.hC.sp.hChs.1.b.pssb..h.psCh............Cbsss.....pshbCC..psbCNp Consensus $\mathbf{8 0} \%$

b

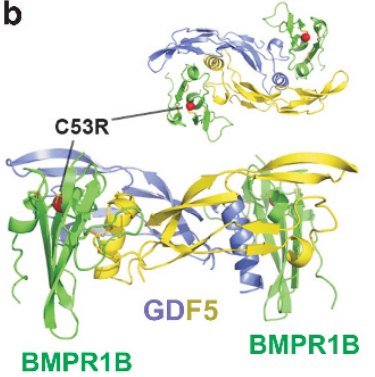

C

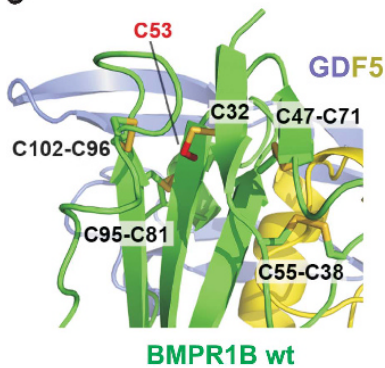

d

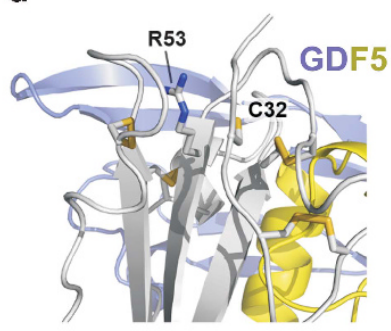

BMPR1B C53R

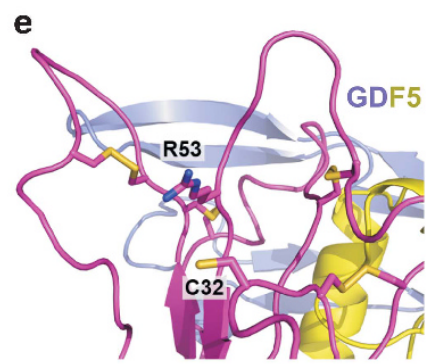

BMPR1B C53R
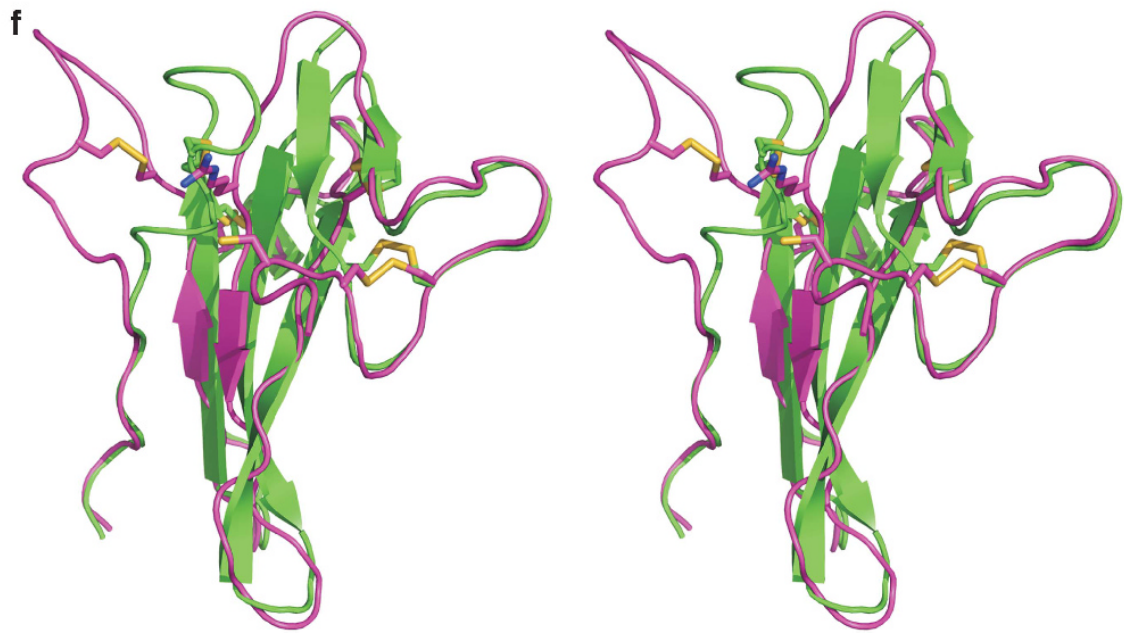
the younger brother was $119 \mathrm{~cm}$ tall $(-6 \mathrm{SD})$ and the older brother was $134 \mathrm{~cm}$ tall $(-5.6 \mathrm{SD})$. The hands of both brothers showed marked shortening of digits and mild radial deviation of fingers with all fingernails being present (Figure $1 \mathrm{Bc}$ and $\mathrm{j}$ ). The fibulae were not palpable. Valgus instability of the ankle joints was evident. The feet were small and broad with all toes being short and functionless with constrictions at their bases and all toenails being present (Figure 1Bd and $\mathrm{k})$.

On X-ray examination, tibiae were shortened but not bowed (Figure $1 \mathrm{Bg}$ and $\mathrm{k}$ ). The fibulae were absent resulting in bilateral dislocation of the tibiotalar joints (Figure $1 \mathrm{Bf}$ and $\mathrm{h}$ ). Bilateral shortened ulnae but no bowing of the radii was observed. Carpal bones were deformed and there were carpal fusions in both affected brothers and additional fusions of the 2nd and 3rd metacarpals in the older brother. Metacarpals were shortened with the absence of 1st metacarpals, and short and deformed proximal and middle phalanges of the fingers were observed with the absence of the proximal phalanx of the index fingers (Figure 1Be and i). In contrast to the affected girl from family $\mathrm{I}$, in both brothers a small proximal phalanx of the thumbs as well as a small middle phalanx of the 5th fingers were present. In the feet, talus, calcaneus, and possible remnants of some ossa navicularia were visible and all metatarsals were present. All distal
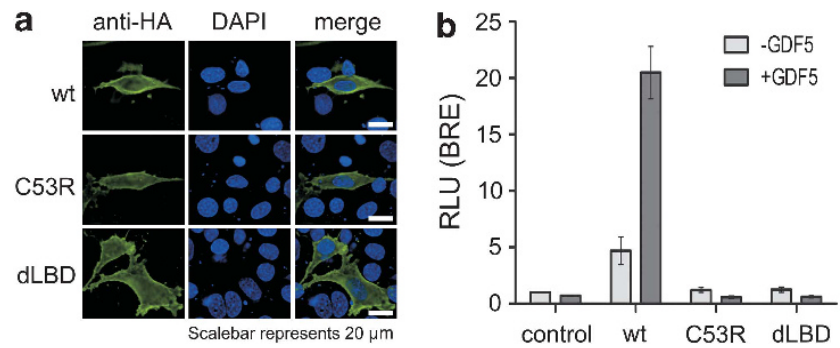

Figure 3 BMPR1B-mutant C53R does not mediate GDF5-dependent signaling. (a) NIH/3T3 cells were transfected with HA-tagged mouse Bmpr $1 \mathrm{~b}$ constructs (wt, C53R or deletion of the LBD (dLBD)). Immunostaining against HA (shown in green) shows that BMPR1Bwt, BMPR1BC53R, and BMPR1BdLBD are located at the cell surface. Cell nuclei are stained with DAPI (shown in blue). Deconvolved wide-field images are shown, the scale bar represents $20 \mu \mathrm{m}$. (b) NIH/3T3 cells were transfected with $50 \mathrm{ng}$ of the BMP responsive reporter pGL3ti-BRE and Renilla normalization vector $\mathrm{pRL}$ TK and $0.5 \mathrm{ng}$ of plasmid vectors expressing either BMPR1B ${ }^{\text {wt }}$, BMPR $1 B^{C 53 R}$, or BMPR $1 B^{\text {dLBD }}$ and co-transfected either with 50 ng of GDF5 or an empty vector control. Results are shown as the relative luciferase activity (RLU) normalized to the relative luciferase activity of cells transfected with the empty vector control. All values are represented as mean \pm SD of triplicate samples. Representative data are shown from three independent experiments. phalanges of the feet were hypoplastic; some mislocalized middle or proximal phalanges seemed to be present (Figure $1 \mathrm{Bf}$ and $\mathrm{h}$ ).

The parents presented with short stature and the father with mild generalized brachydactyly (Supplementary Figure 1; mother: Ba-c; father: $\mathrm{Bd}-\mathrm{f}$ ).

For a summary of phenotypic features and comparison of the patients presented here together with the patient from Demirhan et al, ${ }^{14}$ see Table 1.

\section{Molecular analysis results}

In both families, mutations in GDF5 were excluded and subsequent sequencing of the BMPR1B gene revealed a homozygous singlenucleotide exchange in family I: c.157T $>$ C, p.(C53R), which has not

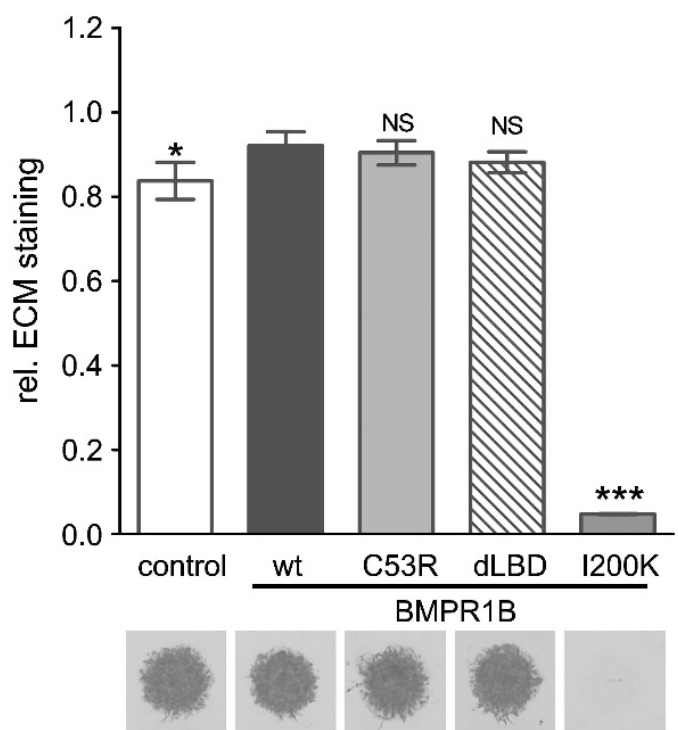

Figure 4 BMPR1B-mutant C53R has no dominant-negative effect on in vitro chondrogenesis. Chicken micromass cultures were infected with either empty viral vector or BMPR1B (wt, C53R, dLBD or the BDA2associated dominant-negative mutation (200K). Micromass cultures were stained with Alcian blue after 7 days of culturing to visualize cartilage formation. Cartilaginous matrix production of cultures expressing, BMPR1B $1 B^{\mathrm{C} 53 \mathrm{R}}$ and BMPR1B ${ }^{\mathrm{dLBD}}$ was not altered in comparison with BMPR1BWT, whereas cultures expressing the BDA2 mutation BMPR1B ${ }^{1200 K}$ exhibited a strong inhibition of chondrogenesis. Alcian blue staining of chicken micromass cultures was quantified by extraction and photometric quantification at $595 \mathrm{~nm}$. All values are represented as mean \pm SD of four replicates from one representative of three independent experiments. Two-tailed Student's $t$-test was performed. Significant differences in comparison with BMPR1BWT are given as ${ }^{*} P<0.05, \quad{ }^{*} P<0.01$, $* * * P<0.001$, or not significant (NS).

Figure 2 Position of the missense mutation p.(C53R) and nonsense mutation p.(W219*) of BMPR1B and proposed pathomechanism. (a) Upper panel: scheme of the mRNA transcript (NM_001256794.1) encoding functional domains of BMPR1B. Lower panel: alignment of the BMPR1B LBD from different species as well as the LBDs from all seven human BMP and TGF- $\beta$-type I receptors. Black lines indicate five conserved disulfide bonds formed by the 10 conserved cysteines. (b) Ribbon representation of the complex of GDF5 bound to BMPR1B (PDB entry 3EVS ${ }^{27}$ ) viewed from the side (lower panel) and from the top (upper panel). The monomer subunits of the dimeric GDF5 are shown in blue and yellow, the LBDs of the two BMPR1B are shown in green. The position of C53, which is exchanged in the BMPR1b-mutant C53R, is marked by a red sphere. (c) Magnification of the disulfide bond network in BMPR1b surrounding C53 (C-atoms colored in red), the cysteine residues forming five disulfide bonds in BMPR1B are indicated. (d) Exchange of C53 by an arginine residue can be accommodated with minimal conformational changes in the structure of BMPR1B if only van der Waals and other geometrical terms are considered (e) As the charged arginine residue introduced by the mutation is completely buried inside the hydrophobic core formed between the $\beta$-strands $1,3,5$ and the C-terminal loop, strong repulsive forces very likely occur, which result from electrostatic mismatch. A short molecular dynamics simulation using the CHARMM27 (MSI Accelrys) force field employing electrostatic energy terms results in unfolding of the upper core structure of BMPR1B. (f) Stereo view of a structural superposition of wild-type BMPR1B (shown in green) and the mutant BMPR1B C53R (shown in magenta) showing that a mutation-induced unfolding in the mutant disrupts a large part of the secondary structure of BMPR1B and alters a large portion of the binding epitope for GDF5.Abbreviations: SP, signal peptide; LBD, ligand-binding domain; TM, transmembrane domain; GS, glycine-serine rich-box; KD, kinase domain; Hs, Homo sapiens; Mm, Mus musculus; Gg, Gallus gallus. 
been reported in the literature to date. The mutation was detected in a heterozygous state in both parents. In family II, a homozygous nonsense mutation in BMPR1B was detected in both affected brothers: c. $657 \mathrm{G}>\mathrm{A}$, p. $\left(\mathrm{W} 219^{*}\right)$, which also has not been reported in the literature to date. This mutation was again detected in a heterozygous state in both parents.

\section{BMPR1B mutations (p. (C53R); p. $\left.\left(\mathrm{W} 219^{*}\right)\right)$ are predicted to cause a} loss of function by different mechanisms

The human BMPPR1B gene is located on chromosome 4 and consists of 10 coding exons. The protein consists of 502 amino acids and contains four functional domains: a LBD, a single-pass transmembrane domain, a glycine-serine-rich-box and a kinase domain (KD; Figure $2 \mathrm{a})$. The nonsense mutation c.657G $>$ A, p. (W219*) introduces a premature stop codon within the KD located in coding exon 6 of 10 of $B M P R 1 B$ and is therefore predicted to activate the nonsensemediated mRNA decay machinery. ${ }^{34}$

The missense mutation c.157T $>\mathrm{C}, \mathrm{p} .(\mathrm{C} 53 \mathrm{R})$ is located in coding exon 2 in the LBD and targets 1 of 10 conserved cysteines normally forming 5 distinct disulfide bridges (C32-C53, C34-C38, C47-71, C81-C95, C96-C102), which are essential for the correct folding of the extracellular domain of the receptor (Figure $2 \mathrm{a}-\mathrm{c}$ ). ${ }^{27} \mathrm{C} 53$ is completely buried inside a hydrophobic core. An exchange of this residue by an arginine also places the polar and charged side chain inside the hydrophobic core, which very likely causes a strong electrostatic repulsion because of its shielding from water and the lack of neutralizing negatively charged residues nearby. This likely causes a partial unfolding of the extracellular LBD of BMPR1B (Figure 2d-f).

BMPR1B ${ }^{\text {C53R }}$ is only partially translocated to the cell membrane $\mathrm{BMPR} 1 \mathrm{~B}$ is a transmembrane receptor mainly located at the cell surface. In order to study whether an altered structure of the LBD caused by the C53R mutation influences its localization, HA-tagged mouse Bmpr1b constructs were expressed in NIH/3T3 cells and stained extracellularly with a HA-specific antibody. Immunofluorescence staining showed that HA-BMPR1B ${ }^{\text {wt }}$ and HA-BMPR1B ${ }^{\mathrm{C} 53 \mathrm{R}}$ as well as an artificially designed construct carrying a deletion of the LBD, HA-BMPR $1 \mathrm{~B}^{\mathrm{dLBD}}$, are all located at the cell membrane (Figure 3a). Notably, fluorescence intensity of the extracellular staining of cells expressing HA-BMPR $1 B^{\mathrm{C} 53 \mathrm{R}}$ was reduced as compared with cells expressing HA-BMPR $1 \mathrm{~B}^{\mathrm{wt}}$ or HA-BMPR1B ${ }^{\text {dLBD }}$, which is not the case when HA-BMPR1B is stained on permeabilized cells, detecting also intracellular proteins (Supplementary Figure 2). Although HA-BMPR1B ${ }^{\mathrm{C} 53 \mathrm{R}}$ is processed and can translocate to the cell surface in our overexpression studies, the efficiency of these processes might be reduced.

\section{GDF5-dependent receptor activation is lost in BMPR1B ${ }^{\mathrm{C} 53 \mathrm{R}}$}

BMPR1B-mediated signaling can be activated by ligands, such as various BMPs and GDF5, which has a very high affinity for BMPR1B. ${ }^{3,27}$

We tested whether BMPR1B ${ }^{\mathrm{C} 53 \mathrm{R}}$ can mediate GDF5-dependent signaling with a BMP responsive luciferase (BRE) reporter assay. NIH/ 3T3 cells were transiently co-transfected with a BRE luciferase reporter and expression constructs of either BMPR1B ${ }^{\mathrm{wt}}$, $B M P R 1 B^{C 53 R}$, or $B M P R 1 B^{\mathrm{dLBD}}$. In the absence of exogenously expressed ligand, a slight activation of the luciferase signal was observed for BMPR1B ${ }^{\text {wt }}$ (Figure 3b). When BMPR1B ${ }^{\text {wt }}$ was coexpressed with GDF5, there was a prominent increase in the luciferase signal, which was not observed in cells co-expressing BMPR1B ${ }^{\mathrm{C} 53 \mathrm{R}}$ and GDF5 (Figure 3b). Similar to BMPR1B ${ }^{\mathrm{dLBD}}$, which lacks the binding site for GDF5, the signal activity of BMPR1B ${ }^{\mathrm{C} 53 \mathrm{R}}$ did not significantly differ from cells, which were transfected with an empty vector control (Figure $3 \mathrm{~b}$ ). Thus, BMPR1B ${ }^{\mathrm{C} 53 \mathrm{R}}$ was not able to mediate BMP signaling induced by GDF5.

\section{BMPR1B mutation C53R has no dominant-negative effect on in vitro chondrogenesis}

We further studied the effect of the mutation C53R on BMPR1B by analyzing its effect on chondrogenic differentiation in vitro using the chicken micromass culture system. ${ }^{35}$ Previously reported point mutations in BMPR1B associated with BDA2 showed a dominantnegative effect on chondrogenesis, when overexpressed in this system. ${ }^{15,21}$ RCASBP(A) virus expressing the chicken orthologs of $\mathrm{BMPR} \mathrm{B}^{\mathrm{wt}}$, BMPR1B ${ }^{\mathrm{C} 53 \mathrm{R}}, \mathrm{BMPR} 1 \mathrm{~B}^{\mathrm{dLBD}}$, and the BDA2-associated dominant-negative mutant BMPR1B ${ }^{\mathrm{I} 200 \mathrm{~K}} \cdot{ }^{15}$ or empty virus as a control were used to infect micromass cultures. After 7 days, extracellular matrix production was evaluated by Alcian blue staining of proteoglycans in the formed cartilaginous matrix (Figure 4). When comparing the degree of chondrogenic differentiation of micromass cultures expressing BMPR1B $1 \mathrm{~B}^{\mathrm{wt}}, \mathrm{BMPR} 1 \mathrm{~B}^{\mathrm{C} 53 \mathrm{R}}$, or $B M P R 1 B^{d L B D}$ with control cultures, no significant differences were observed. As expected, micromass cultures expressing the BDA2associated mutant BMPR1B $\mathrm{B}^{\mathrm{I} 200 \mathrm{~K}}$ hardly displayed any Alcian blue staining, which indicates a strong dominant-negative effect on chondrogenic differentiation (Figure 4). In contrast to the BDA2associated mutation $\mathrm{BMPR} 1 \mathrm{~B}^{\mathrm{I} 200 \mathrm{~K}}$, the $\mathrm{ACD}$-associated mutation $\mathrm{BMPR} 1 \mathrm{~B}^{\mathrm{C} 53 \mathrm{R}}$ seemed rather to constitute a loss-of-function mutant and not eliciting a dominant-negative effect.

\section{DISCUSSION}

All three patients with homozygous BMPR1B mutations reported here show a severe form of limb malformation consisting of bilateral aplasia of the fibula, severe brachydactyly, and fusion of carpal/tarsal bones as similarly seen in other ACDs. The female patient with the c.157T > C, p.(C53R) missense mutation additionally presented with bowing of tibiae and radii, which was not present in the two affected brothers with the c.657G $>$ A, p. (W219*) nonsense mutation. For both mutations, we propose a loss-of-function mechanism. The missense mutation C53R disrupts one of five highly conserved disulfide bridges within the extracellular domain of BMPR1B. The disulfide bonds are important for folding and stability of the protein. ${ }^{27}$ Short molecular dynamics simulation of BMPR1B carrying the C53R mutation employing a force field handling with electrostatic energy terms indicates a severe misfolding of the extracellular domain because of the unbalanced charge of the arginine residue buried into the hydrophobic core of the LBD of BMPR1B, which implicates a loss-of-function pathomechanism. This hypothesis was supported by our overexpression studies where membrane localization of the mutated receptor BMPR1B ${ }^{\mathrm{C} 33 \mathrm{R}}$ was still observed, albeit without activation by the high-affinity ligand GDF5 in reporter gene analysis. It is noteworthy that the point mutation C53R had the same effect as a mutant Bmprlb construct missing the entire LBD. It is possible that at a lower endogenous expression level, the mutated protein is degraded rapidly, as a free cysteine and a missing central disulfide bond might trigger proteasomal degradation during endoplasmic reticulum control (for review see Ellgaard et $a^{36}$ ). Moreover, our data indicate that even receptors with membrane localization can be functionally inactive. This is also supported by our overexpression experiment using the 
micromass culture system, where BMPR $1 \mathrm{~B}^{\mathrm{C} 53 \mathrm{R}}$ had no negative effect on chondrogenic differentiation, unlike the BDA2-associated dominant-negative BMPR1B ${ }^{\text {I200K }}$ mutation. ${ }^{15}$ BMPR1B $^{\text {I200K }}$ might act as a ligand sink by binding BMPs without initiation of signal transduction, whereas BMPR1B ${ }^{\mathrm{C} 53 \mathrm{R}}$ does not bind ligands. Therefore, we propose a loss of function rather than a dominant-negative pathomechanism for the ACD-associated C53R mutation.

The nonsense mutation c.657G $>$ A, p. (W219*) most likely fails to yield functional protein, as the transcripts encoded are likely subject to the nonsense-mediated RNA decay. So far, homozygous mutations in BMPR1B have only once been shown to cause a subtype of ACD with additional genital anomalies caused by a homozygous 8 bp deletion (c.361_368delGGACCTAT), which is located in the extracellular domain of the BMPR1B protein. ${ }^{14}$ This mutation is also proposed to result in a loss of function because of a frameshift and premature stop codon followed by nonsense-mediated RNA decay. ${ }^{14}$ This patient showed uterus hypoplasia and displayed ovarian dysfunction resulting in hypergonadotropic hypogonadism. In contrast, the uterus of our only female patient carrying the homozygous missense mutation c.157T $>$ C, p.(C53R) appeared normal, but ovaries could not be evaluated by ultrasound examination. Therefore, her phenotype does not seem to differ from the other GDF5-associated ACD phenotypes.

Interestingly, heterozygous mutation carriers presented in this paper showed no classical BDA2 phenotype. Only the mother of our female patient with the missense mutation showed a mild form BDA2, whereas the father in family II presented with mild generalized brachydactyly. Moreover, the heterozygous BMPR1B mutation carriers in the family described by Demirhan et a ${ }^{14}$ did not show any signs of brachydactyly. Thus, we propose a loss-of-function pathomechanism for homozygous BMPR1B mutations causing ACD, whereas BDA2 is caused by heterozygous dominant-negative mutations in BMPR1B. ${ }^{15,21}$

These findings widen the phenotypic spectrum of ACD caused by homozygous BMPR1B mutations and may help in ACD diagnosis.

\section{CONFLICT OF INTEREST}

The authors declare no conflict of interest.

\section{ACKNOWLEDGEMENTS}

We thank the family members who participated in this study. Contributions were made possible by DFG funding through the Berlin-Brandenburg School for Regenerative Therapies GSC 203 (UW, AD).

1 Kornak U, Mundlos S: Genetic disorders of the skeleton: a developmental approach. Am J Hum Genet 2003; 73: 447-474.

2 Warman ML, Cormier-Daire V, Hall C et al: Nosology and classification of genetic skeletal disorders: 2010 revision. Am J Med Genet Part A 2011; 155A: 943-968.

3 Nickel J, Kotzsch A, Sebald W, Mueller TD: A single residue of GDF-5 defines binding specificity to BMP receptor IB. J Mol Biol 2005; 349: 933-947.

4 Al-Yahyaee SA, AI-Kindi MN, Habbal O, Kumar DS: Clinical and molecular analysis of Grebe acromesomelic dysplasia in an Omani family. Am J Med Genet Part A 2003; 121A: 9-14.

5 Basit S, Naqvi SK, Wasif N, Ali G, Ansar M, Ahmad W: A novel insertion mutation in the cartilage-derived morphogenetic protein-1 (CDMP1) gene underlies Grebe-type chondrodysplasia in a consanguineous Pakistani family. BMC Med Genet 2008; 9: 102.

6 Douzgou S, Lehmann K, Mingarelli R, Mundlos S, Dallapiccola B: Compound heterozygosity for GDF5 in Du Pan type chondrodysplasia. Am J Med Genet Part $A$ 2008; 146A: 2116-2121.

7 Faiyaz-Ul-Haque M, Ahmad W, Zaidi SH et al: Mutation in the cartilage-derived morphogenetic protein-1 (CDMP1) gene in a kindred affected with fibular hypoplasia and complex brachydactyly (DuPan syndrome). Clin Genet 2002; 61: 454-458.
8 Faiyaz-UI-Haque M, Faqeih EA, Al-Zaidan H, Al-Shammary A, Zaidi SH: Grebe-type chondrodysplasia: a novel missense mutation in a conserved cysteine of the growth differentiation factor 5. J Bone Miner Metab 2008; 26: 648-652.

9 Savarirayan R, White SM, Goodman FR et al: Broad phenotypic spectrum caused by an identical heterozygous CDMP-1 mutation in three unrelated families. Am J Med Genet Part A 2003; 117A: 136-142.

10 Stelzer C, Winterpacht A, Spranger J, Zabel B: Grebe dysplasia and the spectrum of CDMP1 mutations. Pediatr Pathol Molec Med 2003; 22: 77-85.

11 Szczaluba K, Hilbert K, Obersztyn E, Zabel B, Mazurczak T, Kozlowski K: Du Pan syndrome phenotype caused by heterozygous pathogenic mutations in CDMP1 gene. Am J Med Genet Part A 2005; 138: 379-383.

12 Thomas JT, Kilpatrick MW, Lin K et al: Disruption of human limb morphogenesis by a dominant negative mutation in CDMP1. Nat Genet 1997; 17: 58-64.

13 Thomas JT, Lin K, Nandedkar M, Camargo M, Cervenka J, Luyten FP: A human chondrodysplasia due to a mutation in a TGF-beta superfamily member. Nat Genet 1996; 12: 315-317.

14 Demirhan O, Turkmen S, Schwabe GC et al: A homozygous BMPR1B mutation causes a new subtype of acromesomelic chondrodysplasia with genital anomalies. J Med Genet 2005; 42: 314-317.

15 Lehmann K, Seemann P, Stricker S et al: Mutations in bone morphogenetic protein receptor 1B cause brachydactyly type A2. Proc Natl Acad Sci USA 2003; 100: 12277-12282.

16 Curtis D: Heterozygote expression in Grebe chondrodysplasia. Clin Genet 1986; 29. 455-456.

17 Holder-Espinasse M, Escande F, Mayrargue E et al: Angel shaped phalangeal dysplasia, hip dysplasia, and positional teeth abnormalities are part of the brachydactyly C spectrum associated with CDMP-1 mutations. J Med Genet 2004; 41: e78.

18 Everman DB, Bartels CF, Yang Y et al: The mutational spectrum of brachydactyly type C. Am J Med Genet 2002; 112: 291-296.

19 Gutierrez-Amavizca BE, Brambila-Tapia AJ, Juarez-Vazquez $\mathrm{Cl}$ et al: A novel mutation in CDMP1 causes brachydactyly type $C$ with "angel-shaped phalanx". A genotype-phenotype correlation in the mutational spectrum. Eur J Med Genet 2012; 55: 611-614.

20 Polinkovsky A, Robin NH, Thomas JT et al: Mutations in CDMP1 cause autosomal dominant brachydactyly type C. Nat Genet 1997; 17: 18-19.

21 Lehmann K, Seemann P, Boergermann J et al: A novel R486Q mutation in BMPR1B resulting in either a brachydactyly type C/symphalangism-like phenotype or brachydactyly type A2. Eur J Hum Genet 2006; 14: 1248-1254.

22 Seemann P, Schwappacher R, Kjaer KW et al: Activating and deactivating mutations in the receptor interaction site of GDF5 cause symphalangism or brachydactyly type A2. J Clin Invest 2005; 115: 2373-2381.

23 Schwabe GC, Turkmen S, Leschik G et al: Brachydactyly type C caused by a homozygous missense mutation in the prodomain of CDMP1. Am J Med Genet Part A 2004; 124A: 356-363.

24 Goujon M, McWilliam H, Li W et al: A new bioinformatics analysis tools framework at EMBL-EBI. Nucleic Acids Res 2010; 38: W695-W699.

25 Sievers F, Wilm A, Dineen D et al: Fast, scalable generation of high-quality protein multiple sequence alignments using Clustal Omega. Mol Syst Biol 2011; 7: 539.

26 Goodstadt L, Ponting CP: CHROMA: consensus-based colouring of multiple alignments for publication. Bioinformatics 2001; 17: 845-846.

27 Kotzsch A, Nickel J, Seher A, Sebald W, Muller TD: Crystal structure analysis reveals a spring-loaded latch as molecular mechanism for GDF-5-type I receptor specificity. EMBO J 2009; 28: 937-947.

28 Logan M, Tabin C: Targeted gene misexpression in chick limb buds using avian replication-competent retroviruses. Methods 1998; 14: 407-420.

29 Korchynskyi O, ten Dijke P: Identification and functional characterization of distinct critically important bone morphogenetic protein-specific response elements in the Id1 promoter. J Biol Chem 2002; 277: 4883-4891.

30 Hampf M, Gossen M: A protocol for combined Photinus and Renilla luciferase quantification compatible with protein assays. Anal Biochem 2006; 356: 94-99.

31 Hughes SH, Greenhouse JJ, Petropoulos CJ, Sutrave P: Adaptor plasmids simplify the insertion of foreign DNA into helper-independent retroviral vectors. J Virol 1987; 61: 3004-3012.

32 Stricker S, Fundele R, Vortkamp A, Mundlos S: Role of Runx genes in chondrocyte differentiation. Dev Biol 2002; 245: 95-108.

33 Hamburger V, Hamilton HL: A series of normal stages in the development of the chick embryo. 1951. Dev Dyn 1992; 195: 231-272.

34 Kervestin S, Jacobson A: NMD: a multifaceted response to premature translational termination. Nat Rev Mol Cell Biol 2012; 13: 700-712.

35 DeLise AM, Stringa E, Woodward WA, Mello MA, Tuan RS: Embryonic limb mesenchyme micromass culture as an in vitro model for chondrogenesis and cartilage maturation. Methods Mol Biol 2000; 137: 359-375.

36 Ellgaard L, Helenius A: Quality control in the endoplasmic reticulum. Nat Rev Mol Cell Biol 2003; 4: 181-191.

37 Robinson PN, Kohler S, Bauer S, Seelow D, Horn D, Mundlos S: The Human Phenotype Ontology: a tool for annotating and analyzing human hereditary disease. Am J Hum Genet 2008; 83: 610-615. 